\title{
Electron-Hole Distribution and Exciton Condensed Phase Formation in Semiconductor Quantum Wells
}

\author{
A.A. Chernyuk and V.I. Sugakov* \\ Institute for Nuclear Research, NAS of Ukraine \\ 47, Nauki Ave., Kyiv 03680, Ukraine
}

\begin{abstract}
We study the development of ring luminescence of indirect excitons at macroscopical distances from the central excitation spot in quantum well structures. The Landau model for exciton condensation generalized for particles with finite lifetimes in conditions of inhomogeneous excitation is proposed. The transition between the fragmented and continuous rings and the temperature dependence of the effects are considered. The irradiation of the system by two spatially separated laser spots is simulated as well.
\end{abstract}

PACS numbers: 71.35.Lk, 73.21.Fg

\section{Introduction}

In the last years interesting results were obtained for the system of indirect excitons in double quantum wells (QW's). Indirect excitons have long lifetime, because electrons and holes are separated in the space. In AlGaAs and InGaAs double QW structures, a spot of the laser excitation was reported to be surrounded by a concentric bright ring separated from the laser spot by a dark intermediate region [1-4]. The distance between the ring and the spot grew with increasing the intensity of the pumping. This distance significantly exceeded the exciton diffusion length. At low temperatures, the ring broke down into a structure with fragments sited periodically on a macroscopic scale $[1,3]$.

The mechanism of ring appearance was suggested in $[3,4]$ basing on two assumptions: (1) without light irradiation the well is populated with a certain density of electrons; (2) holes are captured by the well with a larger probability than electrons. As the result of charge separation, the irradiated structure divides into two differently charged spatial regions: holes nearby the central spot and electrons far from it. The sharp luminescence ring is formed on the boundary between regions of opposite charges.

*corresponding author; e-mail: sugakov@kinr.kiev.ua 
At present, there are two models explaining the appearance of a fragmented ring in the emission around the laser spot $[5,6]$. According to [5], the presence of excitons on the lowest level stimulates exciton relaxation and transition to this level owing to bosonic character of exciton statistics. In this case, as it is shown in [5], spatial instability and inhomogeneous exciton distribution may emerge. This model has a problem with the correct prediction of the temperature of the ring fragmentation, because in the experiment [1] the fragmentation is observed at higher temperature (or at smaller exciton density) than the theory predicts. The model [6] was based on the traditional phase transition theory, generalized for the case of a non-equilibrium system, in which created particles (excitons) have finite lifetimes. The condensation takes place in real space, not in momentum space at $k=0$, i.e., the condensed phase is not the Bose-Einstein condensate.

The reasons for the application of the traditional phase transition theory for the description of the exciton system are following.

1) There are always composition fluctuations and the disorder sufficiently toughens the criteria for the appearance of quantum coherence effects [7]. As estimates show, for the exciton density of $10^{10} \div 10^{11} \mathrm{~cm}^{-2}$, the wave function loses its coherence due to the scattering on defects at the distances smaller than the distance between excitons.

2) The exciton condensed phase (exciton liquid) is realized in QW's [8], if the distance between wells is not large (according to [8], at $d<1.1 a_{\mathrm{x}}$, where $d$ is the distance between the wells, $a_{\mathrm{x}}$ is the exciton radius). In this case, the binding energy per exciton is negative.

The aim of the present paper is to describe the fragmented structure of exciton luminescence by solving the phenomenological equation for the exciton density, using the free energy of an exciton system. Our approximation allows to take into account inhomogeneity of the system (the generation rate is an essentially inhomogeneous function of coordinates) and to obtain new results, e.g., a description of a transition from a fragmented to a continuous structure of luminescence ring with changing parameters of the system, the irradiation of the system by two spatially separated laser spots, etc. To solve these problems using a stochastic approach [6] would be very complicated.

\section{Model of the system}

The distribution of electrons and holes in a double QW plane is described by diffusion equations [3, 4]. To study the problem of exciton condensation afterwards, we use the results of calculations of the exciton generations rate $G$, obtained in $[3,4,6]$. As it is shown in Refs. [3, 4], this gives rise to that the exciton generation rate $G(\rho)$ has a sharp maximum at a certain distance $\rho$ from the center. This distance departs from the center with increasing the pumping. See [9] for details.

Therefore, we suggest that excitons are created on the ring, where electrons and holes recombine. If the time of the post-excitation establishment of an equilibrium of the electron and hole densities and their binding into excitons is much 
smaller than the time of the equilibration between different areas, the theoretical models applied in the paper for exciton description are following. The equation for the exciton density is under consideration. It is assumed that a local equilibrium is formed in the system due to quick spin-lattice relaxation. Then, the system can be described by the free energy, which depends on the exciton density concerned with inhomogeneity. In that case, the current density, which is in the exciton density equation, can be written in terms of the free energy, which is chosen in the Landau model. The obtained equation is similar to the equations of spinodal decomposition model [10], but finite exciton lifetime and the pumping are taken into account. Thus, a phenomenological equation for the exciton density can be written down as

$$
\frac{\partial n_{\mathrm{x}}}{\partial t}=-\operatorname{div} \boldsymbol{j}+G(\boldsymbol{r})-\frac{n_{\mathrm{x}}}{\tau_{\mathrm{x}}}
$$

where $n_{\mathrm{x}}$ is the exciton density, $G$ is the number of excitons created in a unit area in a unit time (the exciton generation rate), $\tau_{\mathrm{x}}$ is the exciton lifetime, $\boldsymbol{j}$ is the density of the exciton current: $\boldsymbol{j}=-M \nabla \mu$, where $M$ is the exciton mobility, $\mu=\delta F / \delta n_{\mathrm{x}}$ is the chemical potential, $F$ is the free energy. In the Landau model, the free energy is

$$
F\left[n_{\mathrm{x}}\right]=\int \mathrm{d} \boldsymbol{r}\left[\frac{K}{2}\left(\nabla n_{\mathrm{x}}\right)^{2}+f\left(n_{\mathrm{x}}\right)\right],
$$

where the term $\frac{K}{2}\left(\nabla n_{\mathrm{x}}\right)^{2}$ characterizes the energy due to inhomogeneity, the density of the free energy $f$ is

$$
f\left(n_{\mathrm{x}}\right)=\frac{a}{2}\left(n_{\mathrm{x}}-n_{\mathrm{x}}^{\mathrm{c}}\right)^{2}+\frac{b}{4}\left(n_{\mathrm{x}}-n_{\mathrm{x}}^{\mathrm{c}}\right)^{4} .
$$

The parameters $a, b, n_{\mathrm{x}}^{\mathrm{c}}$ in (3) are phenomenological and can be determined from quantum-mechanics calculations or from the experimental data. The attraction between excitons leads to existence of the exciton condensed phase at $a<0(3)$.

After introducing $\sqrt{K /(-a)}, K /\left(M a^{2}\right)$ and $\sqrt{(-a) / b}$ for length, time and concentration units, correspondingly, Eq. (1) becomes [11]:

$$
\frac{\partial n_{\mathrm{x}}}{\partial t}=-\Delta^{2} n_{\mathrm{x}}+\left(3 n_{\mathrm{x}}^{\mathrm{c} 2}-1\right) \Delta n_{\mathrm{x}}-3 n_{\mathrm{x}}^{\mathrm{c}} \Delta n_{\mathrm{x}}{ }^{2}+\Delta n_{\mathrm{x}}{ }^{3}+G-\frac{n_{\mathrm{x}}}{\tau_{\mathrm{x}}} .
$$

In the case of the infinite exciton lifetime Eq. (4) describes the equilibrium states, i.e., two phases with the densities $n_{\mathrm{x}}{ }^{ \pm}=n_{\mathrm{x}}^{\mathrm{c}} \pm 1$, which correspond to the density of the gas phase $\left(n_{\mathrm{x}}^{-}\right)$and condensed one $\left(n_{\mathrm{x}}^{+}\right)$.

\section{Calculation and discussion}

We studied Eq. (4) numerically and analysed its stationary solutions for the exciton density at the parameters $\tau_{\mathrm{x}}=10^{-7} \mathrm{~s}, n_{\mathrm{x}}^{\mathrm{c}}=1.13 \times 10^{10} \mathrm{~cm}^{-2}$. Other parameters (the diffusion coefficient, the pumping $G$, etc.) are presented in [9]. The simulation showed that the excitons condense into a fragmented ring (Fig. 1a). Regions of high density may be attributed to the condensed phase, and regions of low density to the gas phase. Thus, the created fragments are periodically 


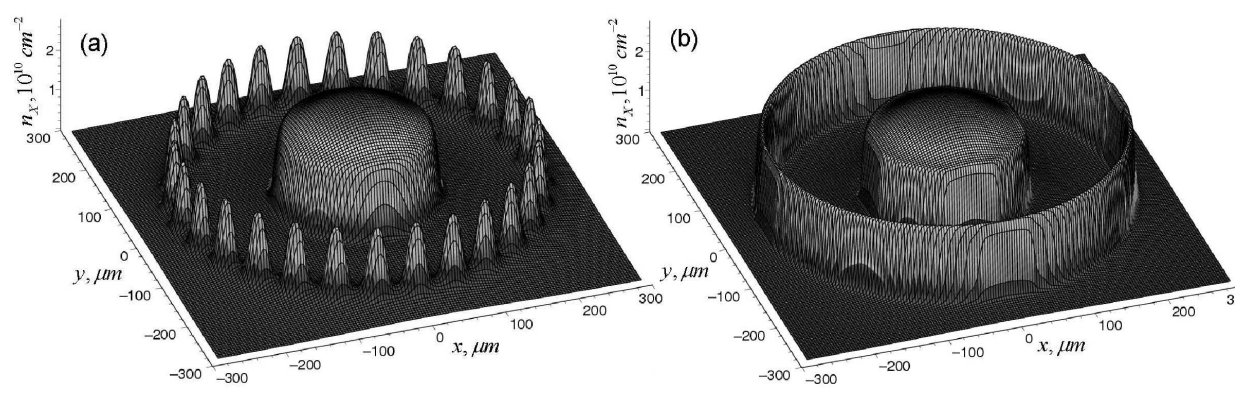

Fig. 1. (a) A fragmented ring of exciton density distribution $n_{\mathrm{x}}(x, y)$. (b) Exciton density at the parameter $K$, which is 5 times smaller than on the left figure.

positioned islands of the exciton condensed phase, the emergence of which is caused by the finite value of the exciton lifetime and the interexciton attraction. The condensed phase consisting of a periodic array of exciton islands corresponds to the fragments observed in $[1,3]$.

The radius of the ring of the condensed phase grows with increasing the pumping rate. With expanding the ring radius, the number of exciton islands increases. They remain of the same size and the distance between them is practically invariant. Similar trends were observed in experiments [1-4].

With decreasing $K$, which determines the surface energy (2), the size of the islands of the condensed excitons diminishes, and the fragmented ring gradually transforms to a continuous one (Fig. 1b).

Let us choose the coefficient $a(3)$ according to the Landau theory: $a(T)=$ $\alpha\left(T_{\mathrm{c}}-T\right)$, where $T_{\mathrm{c}}$ is the critical temperature. The phase transition occurs at the temperature, at which the sign of $a$ changes. The simulations show that with increasing the temperature, the fragmentation of the ring disappears, the intensity of the ring falls down (Fig. 2). With lowering the temperature, the ring

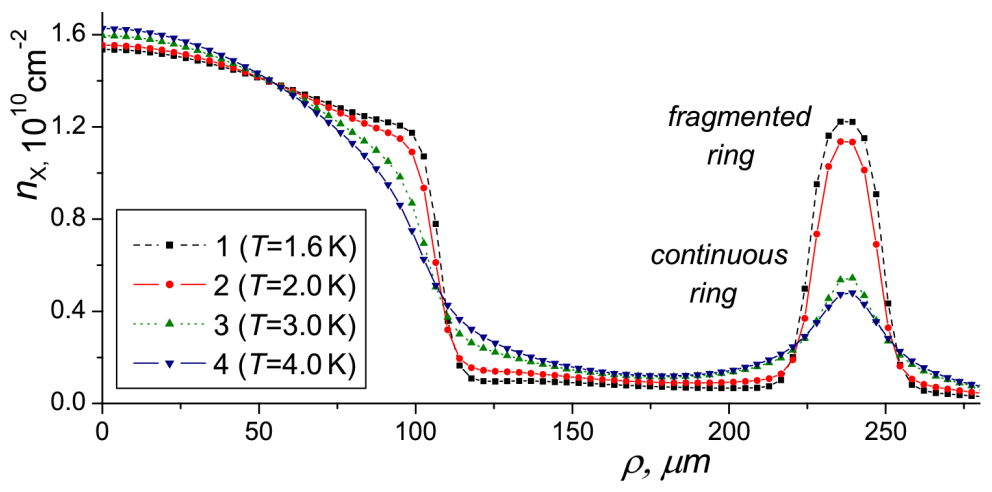

Fig. 2. The radial profile of the exciton density $n_{\mathrm{x}}(\rho)$ at different temperatures. The curves 1 and 2 represent fragmented rings, the curves 3 and 4 are the profiles of low continuous rings. 

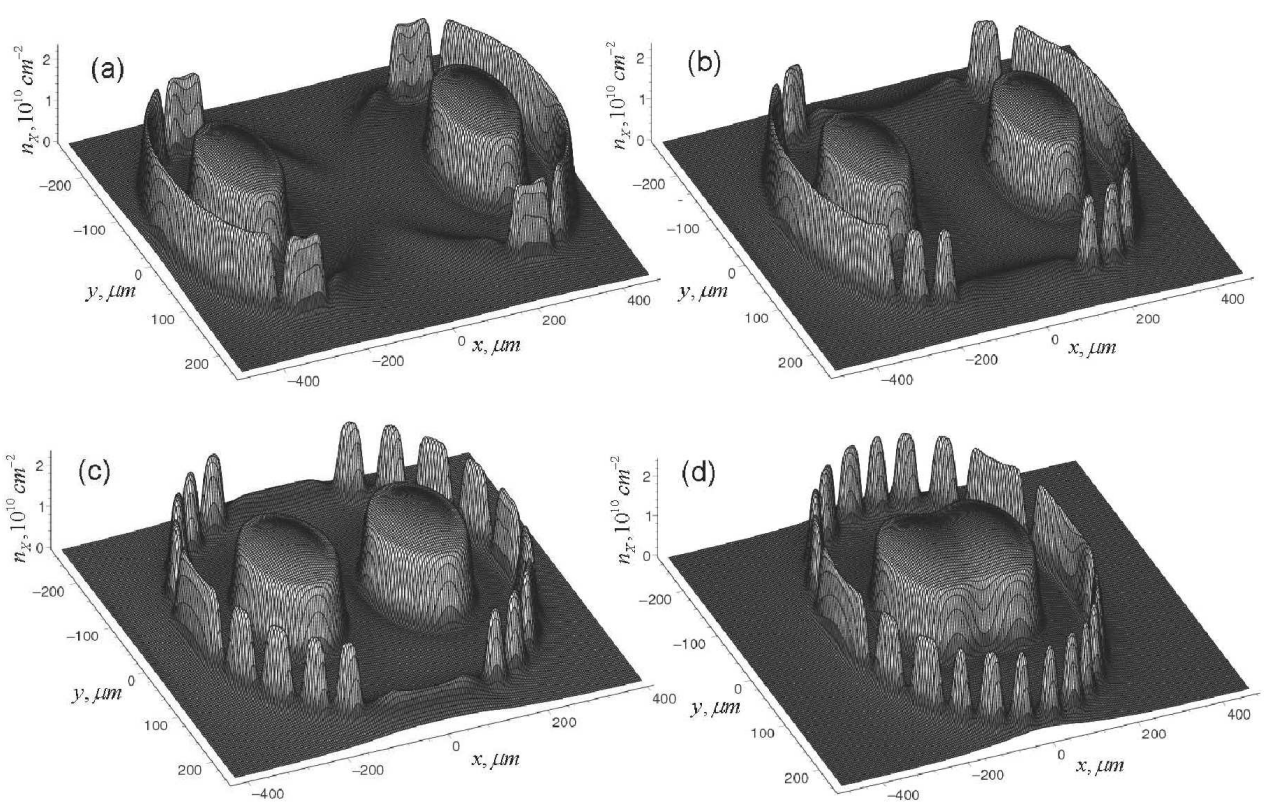

Fig. 3. Exciton density distribution $n_{\mathrm{x}}(x, y)$ in the system irradiated by two laser spots. The distance between the spots centers are: (a) $280 \mu \mathrm{m}$, (b) $200 \mu \mathrm{m}$, (c) $130 \mu \mathrm{m}$, (d) $80 \mu \mathrm{m}$.

remains fragmented. These results agree with the statistical calculations [6] and the experiments [1-4].

The simulation of the irradiation of the system by two spatially separated irradiation sources shows the interaction of the excitation from two spots: the rings become extended in the mutual direction and merge into a common oval-shaped ring at a certain stage of spots approaching (Fig. 3).

\section{Conclusions}

The application of the transitional model of phase transitions for excitons, which have finite lifetimes, allows to explain some experimental results for the system of high density indirect excitons: namely, the appearance of fragmentation in the ring emission outside the laser spot, the transition from a fragmented ring to a continuous one, the irradiation by two laser spots.

\section{Acknowledgments}

The work was supported by INTAS grant No. 03-51-5266. 


\section{References}

[1] L.V. Butov, A.C. Gossard, D.S. Chemla, Nature 418, 751 (2002).

[2] D. Snoke, S. Denev, Y. Liu, L. Pfeiffer, K. West, Nature 418, 754 (2002).

[3] L.V. Butov, L.S. Levitov, A.V. Mintsev, B.D. Simons, A.C. Gossard, D.S. Chemla, Phys. Rev. Lett. 92, 117404 (2004).

[4] R. Rapaport, G. Chen, D. Snoke, S.H. Simon, L. Pfeiffer, K. West, Y. Liu, S. Denev, Phys. Rev. Lett. 92, 117405 (2004).

[5] L.S. Levitov, B.D. Simons, L.V. Butov, Phys. Rev. Lett. 94, 176404 (2005).

[6] V.I. Sugakov, Solid State Commun. 134, 63 (2005).

[7] O.L. Berman, Yu.E. Lozovik, D.W. Snoke, R.D. Coalson, Phys. Rev. B 70, 235310 (2004).

[8] Yu.E. Lozovik, O.L. Berman, JETP 84, 1027 (1997).

[9] A.A. Chernyuk, V.I. Sugakov, arXiv:cond-mat/0508282 2006.

[10] J.W. Cahn, Trans. Metall. Soc. AIME 242, 166 (1968).

[11] V.I. Sugakov, Phase Transit. 75, 953 (2002). 